\title{
Open
}

Review

\section{Cancer predisposition genes: molecular mechanisms and clinical impact on personalized cancer care: examples of Lynch and HBOC syndromes}

Qing WANG *

Laboratory of Constitutional Genetics of Frequent Cancers HCL-CLB; Molecular Biology Platform; Léon Bérard Comprehensive Cancer Center, Lyon, France

Up to $10 \%$ of cancers occur through the inherited mutation of a group of genes called cancer predisposition genes. Individuals who carry a mutant allele of these genes have an increased susceptibility to cancer. A growing number of cancer susceptibility genes are being identified, and the physiopathology of germline mutation-based cancer development is also being elucidated with accumulating clinical and molecular data. More importantly, the identification of familial mutations has become routine practice, which is a perfect example of bench-to-bed translational medicine. Recently, other clinical applications of predisposition genes have been exploited, especially as efficient biomarkers predicting prognosis or response to treatment. Thus, it appears interesting to give an overview of the advances and impacts of predisposition genes in personalized cancer care by taking representative and common cancer syndromes as examples: Lynch syndrome for the first example, which is related to cancer susceptibility, and breast and ovary cancer syndrome for the second example, which involves BRCA deficiency-related targeted therapy.

Keywords: hereditary cancers; cancer predisposition genes; cancer susceptibility; Lynch syndrome; breast and ovary cancer syndrome; biomarkers

Acta Pharmacologica Sinica (2016) 37: 143-149; doi: 10.1038/aps.2015.89; published online 30 Nov 2015

\section{Introduction}

It is now widely established that cancer is a genetic disease that arises through an accumulation of genetic or epigenetic alterations affecting genes that play an essential role in the transformation of normal cells to cancer cells. The majority of cancers are sporadic, result from genetic changes that occur in the tissues of a given organ (somatic alterations). Such genetic alterations are very likely induced by environmental factors such as exposure to UV, chemical agents or viral infections (see IARC monographs: http://www.iarc.fr/). Somatic genomic alterations can also be the consequence of a defective endogenous DNA repair system which is unable to rescue damaged DNA. Sporadic cancers occur generally in aged people, as demonstrated by epidemiology studies that show an increased incidence of cancer in the population aged more than 60 years. Late-onset cancers can reflect a multiple-step process of carcinogenesis across many years with successive inactivation (tumor suppressors) or abnormal activation (oncogenes) of

\footnotetext{
* To whom correspondence should be addressed.

E-mail qing.wang@lyon.unicancer.fr

Received 2015-06-23 Accepted 2015-09-08
}

key cancer genes. Mutations that alter the normal function of those key genes are considered as "driver" mutations allowing for switching the malignant transformation of normal cells.

In contrast to sporadic cancers, up to $10 \%$ of all cancers occur because of inherited genetic defects. The discovery of hereditary cancers is per se a fascinating history of humanity. The first description of inherited cancers was from a pathologist Dr Aldred Warthin who, as early as the late 1890s, established a first pedigree of the "family G" with an impressive aggregation of colon cancers and gynecological cancers in family members throughout four generations ${ }^{[1]}$. It was not until more than 50 years later that Warthin's finding was recalled. In the early 1960s, Dr H Lynch found a similar aggregation of cancers of the digestive system within a family, and this finding triggered his interest in evaluating such familial forms of cancer. From then on and through international collaborations, cancer-prone families were collected and evaluated by Dr H Lynch and colleagues, which led to the identification of hereditary cancer syndromes, especially "Lynch syndrome" (or HNPCC for hereditary non polyposis colorectal cancers) (OMIM \#120435), and "breast and ovarian cancer syndrome" (OMIM \#604370); both are the most common cancer syn- 
dromes. There are also a number of less frequent syndromes with distinctive cancer spectrums ${ }^{[2]}$.

It was logically assumed that familial cancers were caused by inherited genetic elements. Indeed, the molecular basis of familial cancers began to come to light from the early 1990s, due to advanced molecular biological technologies and the genetic linkage approach. It was revealed that genes that are responsible for hereditary cancers, called predisposition genes, follow a loss-of-function model according to Knudson's twohit theory ${ }^{[3]}$. Thus, some number of affected families $(50 \%$ offspring for the autosomal dominant transmission disease) will inherit one allele of a mutated predisposition gene called "germline mutation," which is harbored in every cell of the body. Germline heterozygote mutations of cancer predisposition genes are usually not lethal because their normal function is partially compensated by the normal second allele. However, when the second allele is mutated by somatic environmental or endogenous causes, the given predisposition gene is totally inactivated, leading to the total loss of function and the switch to carcinogenesis in the involved tissues. Therefore, compared with sporadic cases in which two somatic events are needed to inactivate a key gene, germline mutations make the carriers have a much higher risk for cancer development because only one somatic event is required. As a result, hereditary cancers are characterized by 1) early onset of cancer, often younger than 50 years at diagnosis compared with an average age of 60 years in the general population, 2) frequent synchronous and metachronous cancers of the spectrum that reflect a multiple or successive somatic events, and 3) a familial history of cancers through generations in which the cancer types correspond to the syndrome's tumor spectrum.

To a larger extent, all of the genes that are involved in cancer susceptibility would be included in the list of cancer predisposition genes. However, many of them would have a minor involvement in cancer susceptibility, as exemplified by a number of rare variants (rare SNPs) that are revealed from "genome-wild association studys" (GWAS) ${ }^{[4]}$. Their contribution to cancer risk is very likely achieved by a group of SNPs, with each providing a small effect. It is still not clear how those SNPs affect the protein function of the involved genes. Furthermore, the interaction among those SNPs is supposed to be complex. For this review, cancer predisposition genes would only refer to those that have a major risk for cancer following a Mendelian "monogenic" transmission pattern.

Since the early 1990s, the list of high-risk cancer predisposition genes has been continuing to increase. These genes are involved in a variety of cancer syndromes, such as the BRCA1 and $B R C A 2$ genes responsible for hereditary breast and ovary cancer syndrome, DNA mismatch repair genes $M L H 1, M S H 2$, MSH6 and PMS2 for HNPCC syndrome, the APC gene for familial adenomatous polyposis, the MEN1, MEN2 genes for endocrine tumor syndromes, the VHL gene for kidney cancer syndrome, the TP53 gene for Li-Fromeni syndrome, and also the PTEN, NF1 genes ${ }^{[5]}$. Undoubtedly, many other genes remain to be identified, because the cause of cancer heredity for a substantial proportion of the affected families is still unknown. However, each gene is involved in the predisposition in a specific way, in a close relationship with its distinct biological functions, such as DNA repair, cell proliferation, cell death, or signaling pathways. As a result, the physiopathology of each cancer syndrome varies according to which gene is responsible for the malignant transformation and what molecular mechanism is implied. Although there are many questions that are still unanswered in understanding the mechanisms underlying cancer predisposition, for some wellstudied syndromes, accumulated data has allowed for providing a comprehensive scenario, such as in the case of Lynch syndrome. Importantly, besides being responsible for cancer susceptibility, predisposition genes can also act as biomarkers of the response to treatment. Very recently, the predisposition genes $B R C A 1 / B R C A 2$ have been shown to be predictive biomarkers for anti-PARP targeted therapy in ovarian cancer. Thus, this review attempts to outline some of the historical and recent findings on predisposition genes with regard to two aspects: their role in cancer susceptibility, taking Lynch syndrome as an example, and their impact in cancer targeted therapy, involving especially the BRCA genes.

\section{Genetics and clinical impact of predisposition to Lynch syndrome (or HNPCC: Hereditary Non Polyposis Colon Cancer)}

Clinical diagnostics of Lynch and Lynch-related syndrome

The clinical criteria for the diagnosis of Lynch syndrome were initially established by an international consortium referred to as the "Amsterdam criteria," which define a family affected by this syndrome when there are three or more individuals diagnosed with colorectal cancers among first-degree relatives through at least two generations, with at least one patient being diagnosed under the age of 50 years $^{[6]}$. The criteria were later enlarged (Amsterdam II) to include extra-colorectal tumors that are frequently observed in the affected families, $i e$, cancers of the endometrium, ovary, other digestive tract (stomach, small bowl), and biliary and upper urinary tract ${ }^{[6]}$. This disease is transmitted with Mendelian autosomal dominant inheritance. Thus, $50 \%$ of a carrier's offspring would be predisposed to high-risk cancers of the spectrum. Some clinical characteristics appear to be associated with this disease, such as the predominant right-side localization of colon tumors. Compared with the general population, the affected individuals are diagnosed at a younger age (approximately 40 years) but generally have a better outcome.

There exist Lynch-related syndromes such as Muir-Torre syndrome and Turcot syndrome. In addition to the classical Lynch tumor spectrum, the former is characterized by the presence of skin tumors (typically sebaceous neoplasia), and the latter is characterized by brain tumors of glial cell origin. Another particular form of Lynch-related syndrome is called CMMRD (constitutional MMR deficiency syndrome), which is caused by germline homozygous mutations or heterozygous composite mutations that lead to a constitutional inactivation of MMR. This syndrome was first reported in 1999 by our laboratory ${ }^{[7]}$. Since then, approximately 80 families worldwide 
have been described. Clinically, it is characterized by childhood cancers more often of leukemia, brain cancers or very early onset colorectal or endometrial cancers ${ }^{[8]}$.

\section{Lynch syndrome is caused by the inactivation of MMR genes}

Currently, four genes are known to be clearly involved in Lynch syndrome, namely MLH1, MSH2, MSH6 and PMS2. All of them belong to the DNA mismatch repair (MMR) system. Recently, it was found that the EPCAM gene is also associated with a small subset of Lynch patients ${ }^{[9]}$. EPCAM is located upstream of the $\mathrm{MSH} 2$ gene, and the germline genomic deletion of its 3' part results in dysregulation of $\mathrm{MSH} 2$ promoter methylation in somatic tissue. The DNA mismatch repair is well-conserved cellular machinery for correcting errors that are produced during DNA replication. Such errors occurred most frequently in repetitive sequences known as "microsatellites," because of the slippage of DNA polymerase. Although the mechanism of human MMR repair has not yet entirely been understood, schematically, it comprises the following major steps. When a nucleotide is mistakenly paired to the template sequence, or one or several nucleotides are deleted, or one or several extra nucleotides are inserted, the mismatched sequence would be recognized by the MutL protein complex: either MLH1/PMS2 (MutLa) or MLH1/MLH3 (MutL $\beta$ ), followed by recruitment of a MutS complex, either MSH2/MSH6 (MutSa) or MSH2/MSH3 (MutSß). The binding of these complexes to the mismatched sequences further triggers the biochemical reactions with the help of enzymes such as exonucleases to degrade the wrong sequences and DNA polymerase to synthesize the correct sequence. This simplified description illustrates the essential role of MLH1, MSH2, MSH6 and PMS2 genes in DNA mismatch repair. Inactivation of any one of them leads to the dysfunction of the system to a variable extent. The MLH1 and MSH2 genes presumably cause more serious damage because they are key partners of both protein complexes. Indeed, among the patients with Lynch syndrome, mutations in the MLH1 and MSH2 genes are predominant and are responsible for up to $80 \%$ of the cases. The MSH6 gene mutation accounts for $10 \%$ to $15 \%$ of the cases. Mutations in PMS2 appear to be even rarer and are estimated to be less than $5 \%$. However, the mutation frequency for the PMS2 gene is very likely underestimated because the mutation detection is complicated by the presence of a number of pseudogenes that share high sequence homology with the authentic PMS2 gene.

\section{Microsatellite instability (MSI) phenotype}

Microsatellites are small DNA elements of repetitive sequences, either mononucleotide repeats (SSR: simple sequence repeat) or repeats of 2 to 10 nucleotides (STR: short tandem repeats or VNTR: variable number tandem repeats). They are abundantly present in the genome of eukaryotes and occur nearly every 50 kilobases throughout the genome. In addition to the fact that they are very polymorphic making them perfect biomarkers for population biological or epidemiological studies, they are also sensitive targets of DNA polymerase slippage during the course of replication ${ }^{[10,11]}$. Such slippage results in the deletion or insertion of repetitive motives, and they are corrected, in a normal situation, by the MMR system. In contrast, unrepaired deletion/insertion errors lead to changes in the sequence lengths of the given microsatellites, which can be revealed by PCR (polymerase chain reaction) followed by electrophoresis analysis with acrylamide gel. If such variations are detected in a certain proportion of microsatellites in the tumor tissue compared to the normal tissue of the same individual, then this tumor is considered to have a "microsatellite instability" phenotype, which suggests a defective MMR. To evaluate the degree of microsatellite instability in a more standard way, different panels of microsatellites have been proposed ${ }^{[12,13]}$. Currently, a 5-marker panel, which is commercialized by Promega as a fluorescent multiplex assay, is more commonly used ${ }^{[14]}$. Because they are all almost monomorphic markers, there is no need for parallel testing of normal tissue from each patient. A tumor showing instability with more than two markers is interpreted as MSI-High (MSI-H), and a tumor without instability of any of the markers is considered to be microsatellite stable (MSS).

The MSI-H phenotype is caused by defective MMR in the tumor by different mechanisms. Approximately $15 \%$ of the colorectal MSI-H tumors are due to age-related somatic hypermethylation of the MLH1 promoter $^{[15]}$. The remaining MSI-H tumors were, until recently, believed to be the indication of the presence of a germline mutation in the context of Lynch or Lynch-related syndromes. However, recent studies have shown that a small subset of those MSI-H tumors can be the consequence of a pure somatic MMR inactivation through two somatic hits ${ }^{[16,17]}$. Still, because the majority of the MSI-H tumors are germline-related, the testing for the MSI-H phenotype combined with immunostaining for a loss-of-expression of one of four MMR proteins (MLH1, MSH2, MSH6 and PMS2) is considered to be an efficient way to screen for Lynch or related syndromes.

\section{"Mutator phenotype" and Lynch syndrome}

Many studies have been conducted to attempt to determine how a defective DNA mismatch repair system provokes the occurrence of colorectal, endometrial and other tumors in germline mutation carriers. Experiments with cell lines and in bacteria and yeast have shown that cells with a deficient MMR display a highly increased spontaneous mutation rate across the genome and throughout cell division. Spontaneous mutations do occur in normal somatic cells that are estimated to have $1.4 \times 10^{-10}$ nucleotide/cell per division, which corresponds approximately to one mutant gene during an individual's lifetime. Such mutations become eventually tolerated SNPs. However, MSI-H tumor cells can contain as many as $1 \times 10^{5}$ mutations. The high mutation rate that is observed in cancers has been described as the "mutator phenotype ${ }^{1[18]}$, which is induced by endogenous and exogenous factors such as MMR deficiency. An increased mutation rate constitutes an early stage of cancer development. At this stage, a number of genes are likely affected in a random way, although, as in MSI-H 
cells, repetitive sequences are preferentially affected resulting in a "frameshift mutation" signature ${ }^{[19]}$. As a consequence, such a high rate yet random mutations would affect, even "by chance", the coding sequences of oncogenes or tumor suppressors. Once mutated, these key cancer genes confer cancer cells a selective advantage and escape the normal control of proliferation or apoptosis and lead eventually to the cancer cell clonal expansion. Some target genes were indeed found to be associated with Lynch syndrome. The transforming growth factor $\beta$ receptor type 2 gene (TGF $\beta R 2$ ) appeared to be an important target since up to $90 \%$ of the MSI-H colorectal tumors carry a "frameshift mutation" in an A10 mono-repeat sequence of the gene. TGF $\beta$ gene inhibits the growth of colon epithelial cells. When the receptor is inactivated, growth inhibition cannot be accomplished resulting in deregulated cell proliferation. Insulin-like growth factor II receptor (IGFRII) and BAX genes are two other likely targets, because they also contain frequent "frameshift" mutations in their coding sequences. IGFRII regulates TGF receptors and BAX exerts its functions in apoptosis. A number of other genes are also likely targeted, although with less frequency ${ }^{[20]}$. Altogether, the accumulated mutations result in the loss-of-function of these key genes and, in turn, switch and accelerate the development of cancers.

\section{Molecular mechanisms underlying MMR gene inactivation}

Although not defined as classical tumor suppressors but instead as "caretaker genes", MMR genes are inactivated following Knudson's two-hit pattern: one allele by inherited germline mutation and the other by somatic mutation in affected tissues.

The germline inactivations are predominantly truncating mutations that interrupt the synthesis of a protein, including nonsense mutations (the creation of a stop codon by the replacement of a nucleotide), frameshift mutations (small deletions/insertions that lead to the modification of the reading frame and a premature stop codon), genomic gross deletion or insertion and rare complex mechanisms such as genomic inversion ${ }^{[21]}$. Intronic mutations are also involved, especially at the well-conserved splicing sequences ${ }^{[22]}$. However, explorations of intronic mutations being not exhaustive, there are certainly unrevealed causative mutations located in non-coding sequences. Indeed, exon-skipping alterations have been identified in some patients by RNA analysis, with no genomic mutation found in the coding sequences and splicing sites (unpublished data). In addition to genomic alterations, epimutations could also play a role as germline alterations. Nevertheless, this mechanism appears to be more complex. Given the methylation reprogramming process during germ-cell maturation and gametogenesis in mammalian development, an unstable inheritance of methylation patterns can be expected. Germline methylation of MLH1 promoter could be involved in less than $10 \%$ of early onset patients with colorectal cancer ${ }^{[23]}$, with the susceptibility for those patients to develop synchronous and metachronous tumors of the spectrum, because the germline hypermethylation of MLH1 promoter silences the gene expression, mimicking germline genomic alteration. If, in general, truncating mutations are straightforwardly considered to be deleterious mutations, the causalities for nonsynonymous missense mutations are sometimes very difficult to define, because such mutations affect only one nucleotide, leading to an amino acid change without disrupting the protein synthesis. Many parameters should be considered when evaluating whether a given missense mutation tends to alter the protein's normal function, such as evolutionary conservation through species, localization in a functional domain, the biochemical change between the original and replaced amino acid, and the exclusion of a known polymorphism. Clinical, familial and tumor features constitute also a fundamental basis for the interpretation of a given missense mutation's biological significance, including the consistency with the tumor spectrum, the co-segregation of mutant alleles with the disease, and the MSI-H phenotype with probably the loss of expression of a given MMR protein. To give an objective and practical guideline for genetic laboratories and genetic consultants, a 5-level classification of missense mutations has been proposed $^{[24]}$. A large number of missense mutations have thus been classified collectively by different collaborative consortiums ${ }^{[25]}$

The mechanisms of second-hit mutations was less intensively studied because of limited accessibility to tumor samples in the affected patients. In fact, the affected members of a given family were often treated prior to the genetic investigation, and the samples were not necessarily conserved in the adapted condition. However, studies conducted on tumors from Lynch patients revealed that a loss of heterozygosity (LOH), a second mutation in the involved gene and MLH1 promoter hypermethylation are the major causes of somatic mutations in tumors, and each accounts for approximately $30 \%$. Recently developed next-generation sequencing (NGS) has made it possible to analyze simultaneously MMR genes using a small amount of tumor DNA. With more MSI-H tumors tested, somatic events in tumors that are associated with Lynch syndrome can be studied more precisely.

\section{Management of the affected families}

The major aim of screening for mutation of cancer predisposition genes is to take in charge of affected families. In accordance with preventive medicine, this healthcare of the highrisk population is realized by a well-organized multidisciplinary network, and in many countries, it is under the control of specified regulations. The prescriptions usually come from genetic consultations in which geneticists make an enquiry about familial and personal histories of cancers with the help of a pedigree, and they suggest, according to diagnostic criteria, molecular analysis to identify the genetic alterations that are responsible for inherited familial cancers (initial investigation). After a mutation is identified for a given family, predictive testing can be suggested to asymptomatic family members to determine whether they are mutation carriers. For carriers who are at high risk of cancer, adapted clinical surveillance will be carried out typically with regular colonoscopy for 
the detection of colon cancers and transvaginal ultrasound examinations for the detection of endometrial cancer. In some situations, prophylactic treatment will appear to be helpful or necessary. More detailed clinical follow-up can be found in the revised surveillance guidance ${ }^{[26]}$. Cancer risk in mutation carriers varies largely with cancer syndromes and the involved predisposition genes. The lack of a correlation between genotype and phenotype and the variable penetrance (the expressivity of the mutant gene) render the risk estimation very challenging. Indeed, for patients with Lynch syndrome, carriers of the same mutation can have different clinical manifestations even within the same family, and histologically similar malignancies can be caused by a variety of mutations of any one of the MMR genes. Organ-related cancer risk in mutation carriers have been evaluated by some studies ${ }^{[27-29]}$, which are useful in genetic counseling to set up appropriate clinical surveillance. However, the mutation penetrance is believed to be associated with the individual's genetic background through "modifier" SNPs whose interaction with predisposition genes is assumed to regulate the expressivity of the mutant genes ${ }^{[30]}$.

\section{Predisposition genes as biomarkers in cancer treatment}

In addition to high-risk prediction for hereditary cancers, predisposition genes can also be used as prognostic or predictive response biomarkers for sporadic cancers, such as in the case of MMR gene inactivation. In fact, MSI-H tumors are associated with a better outcome compared with MSS tumors ${ }^{[31]}$, with a lower metastasis rate even for patients with an advanced stage of the disease ${ }^{[32]}$. Additionally, deficient MMR is a biomarker for colon cancer treatment. Many studies have suggested that there is no benefit to adjuvant 5-FU chemotherapy for stage II patients with MSI-H tumors ${ }^{[33]}$. Also, recent studies have indicated that the MSI-H phenotype appears to be a predictive response biomarker for treatment with oxaliplatin and irinotecan ${ }^{[34]}$.

Importantly, the emergence of the predisposition genes' roles as biomarkers for targeted cancer therapy has been highlighted with the predictive value of the BRCA1/BRCA2 gene mutations in the response to anti-PARP treatment.

\section{BRCA1/2 gene mutation and predisposition to inherited breast} and ovary cancer syndrome (HBOC)

The $B R C A$ genes are also involved in normal cell DNA repair machinery. Different from MMR, they participate in the repair of double-strand breaks (two strands of DNA are simultaneously broken) by homologous recombination ${ }^{[35]}$. Specifically, BRCA1 combines with other tumor suppressors, DNA damage sensors, and signal transducers to form a large multi-subunit protein complex known as the BRCA1-associated genome surveillance complex (BASC) ${ }^{[36]}$. The physiopathology of HBOC is quite similar to Lynch syndrome. When damaged DNA fails to be repaired, genomic alterations are thus accumulated, which leads to genomic instability. Such increased mutation rate results eventually in the involvement of cancer genes. So far, BRCA1 and BRCA2 genes are two genes that are known to be responsible for HBOC with an autosomal dominant inheri- tance. Germline mutation carriers have a high risk of developing breast cancer and ovarian cancer (approximately $80 \%$ and $50 \%$, respectively $)^{[37]}$. Germline mutations are characterized predominately by protein truncating mutations, including nonsense or frameshifts due to the deletion or insertion of small sequences.

\section{Anti-PARP treatment and BRCA genes}

Poly-ADP ribose polymerase genes (PARP1 and PARP2) encode proteins that play an essential role in the repair of single-strand breaks in DNA. Pharmacological PARP inhibition has been aimed at preventing cells from single-stranded damage repair that is produced by environmental exposure or chemotherapy. Unpaired single strand damage tends most likely to convert to double-strand breaks during replication. Such overwhelmed damage could saturate the repair machinery and bring eventually the cancer cells to death. The use of PARP inhibitors in the early $1980 \mathrm{~s}^{[38,39]}$ showed increased sensitivity to chemo and radiotherapy ${ }^{[40]}$. Accumulated doublestrand breaks depend on BRCA for their repair. In the absence of BRCA, the dysfunction of the repair system will trigger cell death. Such a "synthetic lethality" targets two genetic pathways, by disrupting both types of DNA repair, and makes the carriers of the germline BRCA gene mutation have increased sensitivity to PARP inhibitors. In 2007, the result of the first clinical phase 1 trial was presented at the American Society of Clinical Oncology (ASCO) annual meeting and published later ${ }^{[41]}$; this trial showed the best response to Lynparza (olaparib) treatment in patients who carry the $B R C A$ germline mutation. After a series of phase I to III randomized trials with different anti-PARP agents and with or without BRCA mutations ${ }^{[42]}$, at the end of 2014, the FDA approved the use of Lynparza in ovarian cancer patients with BRCA deficiency.

\section{BRCAness phenotype}

The term BRCAness was designated for sporadic breast or ovary cancers without the BRCA germline mutation but having a behavior similar to the $B R C A$ germline mutation caused tumors. BRCA-like behavior has been described based on clinical and molecular features, such as a high rate of response to platinum-based treatment and an improved overall survival rate $^{[43]}$. Many mechanisms that reduce BRCA1/2 function and result in BRCA-like behavior have been identified, including BRCA1 promoter methylation and the loss of function of genes participating in homologous recombination like the RAD51, ATM, ATR, CHK1, CHK2, FANCD2, and FANCA genes ${ }^{[44-47]}$. Of note, many of these genes are involved in cancer susceptibility. A loss of function of the suppressor gene, PTEN, another cancer predisposition gene involved in Cowden syndrome (OMIM \#158350), has been shown to yield BRCA-like behavior, which is more common in breast and prostate cancers ${ }^{[48]}$. Increased PARP inhibition susceptibility was shown in a series of cell lines with PTEN mutation or haploinsufficiency, confirmed in xenograft experiments using the PARP inhibitor olaparib. There is also clinical evidence that olaparib may have a therapeutic utility in PTEN-deficient endometrioid 
endometrial cancer ${ }^{[49]}$. These studies provide evidence that PTEN loss of function is a potential predictive biomarker of PARP inhibitor responsiveness. However, it is still challenging to identify BRCAness sporadic tumors. Standardized methods need to be developed to evaluate the effectiveness of PARP inhibitor in those tumors.

\section{Conclusions and perspectives}

Cancer arises through complex genetic pathways that implicate a number of genes, which are either drivers or helpers, in the malignant transformation of normal cells. Cancer predisposition genes play an essential role in this process. If over decades their involvement was basically focused on familial inheritance, then emerging evidence strongly suggests that there are many functionalities that remain to be explored. Their role as an indication of a high risk of cancer allows individuals who carry a familial mutation to receive appropriate follow-up and allows non-mutation carriers to be discharged from an intensive surveillance protocol. Their role as response predictive biomarkers helps to select the patients for whom a given targeted therapy appears to have more benefit. All this makes such predisposition genes important actors in personalized cancer care. Moreover, because MMR genes and BRCA genes are involved in the repair system, which is a key function to guarantee the genomic integrality, a deeper understanding of their involvement in cancer initiation and progression is of great interest. Other features may also have high potential importance. For example, MMR- and BRCAdeficient tumors display a high level of lymphocyte infiltration $^{[50,51]}$, reflecting an active immunoreaction in the tumors. An increased mutation rate induced by DNA repair deficiency is believed to be the cause, since altered proteins could be considered to be foreigners by the immune system. In accordance with rapidly advanced immunotherapies, the mutationinduced immunoreaction could well be a new horizon for predisposition gene studies.

\section{References}

1 Warthin AS. Heredity with reference to carcinoma. Arch Intern Med 1913; 12: 546-55.

2 Garber JE, Offit K. Hereditary cancer predisposition syndromes. J Clin Oncol 2005; 23: 276-92.

3 Knudson A. Mutation and cancer: statistical study of retinoblastoma. Proc Natl Acad Sci USA 1971; 68: 820-3.

4 Chung CC, Chanock SJ. Current status of genome-wide association studies in cancer. Hum Genet 2011; 130: 59-78.

5 Lynch HT, Fusaro RM, Lynch J. Hereditary cancer in adults. Cancer Detect Prev 1995; 19: 219-33.

6 Vasen HF, Watson P, Mecklin JP, Lynch HT. "New clinical criteria for hereditary nonpolyposis colorectal cancer (HNPCC, Lynch syndrome) proposed by the International Collaborative group on HNPCC". Gastroenterology 1999; 116: 1453-6.

7 Wang Q, Lasset C, Desseigne F, Frappaz D, Bergeron C, Navarro C, et al. Neurofibromatosis and early onset of cancers in hMLH1-deficient children. Cancer Res 1999; 59: 294-7.

8 Wimmer K, Kratz CP, Vasen HF, Caron O, Colas C, Entz-Werle N, et al. Diagnostic criteria for constitutional mismatch repair deficiency syndrome: suggestions of the European consortium 'care for CMMRD'
(C4CMMRD). J Med Genet 2014; 51: 355-65.

9 Ligtenberg MJ, Kuiper RP, Chan TL, Goossens M, Hebeda KM, Voorendt $\mathrm{M}$, et al. Heritable somatic methylation and inactivation of MSH2 in families with Lynch syndrome due to deletion of the 3 ' exons of TACSTD1. Nat Genet 2009; 41: 112-7.

10 Levinson G, Gutman GA. High frequencies of short frameshifts in poly-CA/TG tandem repeats borne by bacteriophage $\mathrm{m} 13$ in Escherichia coli k-12. Nucleic Acids Res 1987; 15: 5323-38.

11 Ellegren $\mathrm{H}$. Microsatellite mutations in the germline: implications for evolutionary inference. Trends Genet 2000; 16: 551-8.

12 Eshleman JR, Markowitz SD. Microsatellite instability in inherited and sporadic neoplasms. Curr Opin Oncol 1995; 7: 83-9.

13 Boland CR, Thibodeau SN, Hamilton SR, Sidransky D, Eshleman JR, Burt RW, et al. National Cancer Institute Workshop on Microsatellite Instability for cancer detection and familial predisposition: development of international criteria for the determination of microsatellite instability in colorectal cancer. Cancer Res 1998; 58: 5248-57.

14 Suraweera N, Duval A, Reperant M, Vaury C, Furlan D, Leroy K, et al. Evaluation of tumor microsatellite instability using five quasimonomorphic mononucleotide repeats and pentaplex PCR. Gastroenterology 2002; 123: 1804-11.

15 Newton K, Jorgensen NM, Wallace AJ, Buchanan DD, Lalloo F, McMahon RF, et al. Tumour MLH1 promoter region methylation testing is an effective prescreen for Lynch Syndrome (HNPCC). J Med Genet 2014; 51: 789-96.

16 Geurts-Giele WR, Leenen CH, Dubbink HJ, Meijssen IC, Post E, Sleddens HF, et al. Somatic aberrations of mismatch repair genes as a cause of microsatellite unstable cancers. J Pathol 2014; 234: 548-59.

17 Joly MO, Attignon V, Saurin JC, Desseigne F, Leroux D, Martin-Denavit T, et al. Somatic MMR gene mutations as a cause for MSI-H sebaceous neoplasms in Muir-Torre Syndrome-Like patients. Hum Mutat 2015; 36: $292-5$.

18 Loeb LA. A mutator phenotype in cancer. Cancer Res 2001; 61: 3230-9.

19 Mori Y, Yin J, Rashid A, Leggett BA, Young J, Simms L, et al. Instabilotyping: comprehensive identification of frameshift mutations caused by coding region microsatellite instability. Cancer Res 2001; 61: 6046-9.

20 Kawaguchi M, Banno K, Yanokura M, Kobayashi Y, Kishimi A, Ogawa S, et al. Analysis of candidate target genes for mononucleotide repeat mutation in microsatellite instability-high (MSI-H) endometrial cancer. Int J Oncol 2009; 35: 977-82.

21 Auclair J, Leroux D, Desseigne F, Lasset C, Saurin JC, Joly MO, et al. Novel biallelic mutations in MSH6 and PMS2 genes: gene conversion as a likely cause of PMS2 gene inactivation. Hum Mutat 2007; 28 : 1084-90.

22 Auclair J, Busine MP, Navarro C, Ruano E, Montmain G, Desseigne F, et al. Systematic mRNA analysis for the effect of MLH1 and MSH2 missense and silent mutations on aberrant splicing. Hum Mutat 2006; 27: 145-54.

23 Auclair J, Vaissière T, Desseigne F, Lasset C, Bonadona V, Giraud S, et al. Intensity-dependent constitutional MLH1 promoter methylation leads to early onset of colorectal cancer by affecting both alleles. Intensity-dependent constitutional MLH1 promoter methylation leads to early onset of colorectal cancer by affecting both alleles. Genes Chromosomes Cancer 2011; 50: 178-85.

24 PIon SE, Eccles DM, Foulkes WD, Genuardi M, Greenblatt MS, Hogervorst FBL, et al. Sequence variant classification and reporting: recommendations for improving the interpretation of cancer 
susceptibility genetic test results. Hum Mut 2008; 29: 1282-91.

25 Grandval P, Fabre AJ, Gaildrat P, Baert-Desurmont S, Buisine MP, Ferrari A, et al. UMD-MLH1/MSH2/MSH6 databases: description and analysis of genetic variations in French Lynch syndrome families. Database. Vol. 2013, doi:10.1093/database/bat036.

26 Vasen HFA, Blanco I, Aktan-Collan K, Gopie JP, Alonso A, Aretz S, et al. Revised guidelines for the clinical management of Lynch syndrome (HNPCC): recommendations by a group of European experts. Gut 2013 62: 812-23.

27 Dunlop MG, Farrington SM, Carothers AD, Wyllie AH, Sharp L, Burn J, et al. Cancer risk associated with germline DNA mismatch repair gene mutations. Hum Mol Genet 1997; 6: 105-10.

28 Alarcon F, Lasset C, Carayol J, Bonadona V, Perdry H, Desseigne F, et al. Estimating cancer risk in HNPCC by the GRL method. Eur J Hum Genet 2007; 15: 831-6.

29 Bonadona V, Bonaïti B, Olschwang S, Grandjouan S, Huiart L, Longy $\mathrm{M}$, et al. Cancer risks associated with germline mutations in MLH1, MSH2, and MSH6 genes in Lynch syndrome. JAMA 2011; 305: 2304-10.

30 Talseth-Palmer BA, Wijnen JT, Grice DM, Scott RJ. Genetic modifiers of cancer risk in Lynch syndrome: a review. Familial Cancer 2013; 12 : 207-16.

31 Lothe RA, Peltomäki P, Meling GI, Aaltonen LA, Nyström-Lahti $M$, Pylkkänen L, et al. Genomic instability in colorectal cancer: relationship to clinicopathological variables and family history. Cancer Res 1993; 53: 5849-52.

32 Buckowitz A, Knaebel HP, Benner A, Bläker H, Gebert J, Kienle P, et al. Microsatellite instability in colorectal cancer is associated with local lymphocyte infiltration and low frequency of distant metastases. $\mathrm{Br} \mathrm{J}$ Cancer 2005; 92: 1746-53.

33 Sinicrope FA. DNA mismatch repair and adjuvant chemotherapy in sporadic colon cancer. Nat Rev Clin Oncol 2010; 7: 174-7.

34 Yacoub G, Nagalla S, Aklilu M. Oncologic management of hereditary colorectal cancer. Clin Colon Rectal Surg 2012; 25: 118-22.

35 Powell SN, Kachnic LA. Roles of BRCA1 and BRCA2 in homologous recombination, DNA replication fidelity and the cellular response to ionizing radiation. Oncogene 2003; 22: 5784-91.

36 Wang Y, Cortez D, Yazdi P, Neff N, Elledge SJ, Qin J. BASC, a super complex of BRCA1-associated proteins involved in the recognition and repair of aberrant DNA structures. Genes Dev 2000; 14: 927-39.

37 Mavaddat N, Peock S, Frost D, Ellis S, Platte R, Fineberg E, et al. Cancer risks for BRCA1 and BRCA2 mutation carriers. Results from prospective analysis of EMBRACE. J Natl Cancer Inst 2013; 105: 812-22.

38 Durkacz BW, Omidiji O, Gray DA, Shall S. (ADP-ribose)n participates in DNA excision repair. Nature 1980; 283: 593-6.

39 Borek C, Morgan WF, Ong A, Cleaver JE. Inhibition of malignant transformation in vitro by inhibitors of poly(ADP-ribose) synthesis. Proc Natl Acad Sci U S A 1984; 81: 243-7.

40 Thraves PJ, Mossman KL, Brennan T, Dritschilo. Differential radiosensitization of human tumour cells by 3 -aminobenzamide and benzamide: inhibitors of poly(ADP-ribosylation). Int J Radiat Biol Relat Stud Phys Chem Med 1986; 50: 961-72.
41 Fong PC, Boss DS, Yap TA, Tutt A, Wu P, Mergui-Roelvink M, et al. Inhibition of poly(ADP-ribose) polymerase in tumors from BRCA mutation carriers. N Engl J Med 2009; 361: 123-34.

42 Liu JF, Konstantinopoulos PA, Matulonis UA. PARP inhibitors in ovarian cancer: Current status and future promise. Gynecol Oncol 2014; 133: 362-9.

43 Tan DS, Rothermundt C, Thomas K, Bancroft E, Eeles R, Shanley S, et al. "BRCAness" syndrome in ovarian cancer: a case-control study describing the clinical features and outcome of patients with epithelial ovarian cancer associated with BRCA1 and BRCA2 mutations. J Clin Oncol 2008; 25: 5530-6.

44 McCabe N, Turner NC, Lord CJ, Kluzek K, Bialkowska A, Swift S, et al. Deficiency in the repair of DNA damage by homologous recombination and sensitivity to poly(ADP-ribose) polymerase inhibition. Cancer Res 2006; 66: 8109-15.

45 Long KC, Kauff ND. Hereditary ovarian cancer: recent molecular insights and their impact on screening strategies. Curr Opin Oncol 2011; 23: 526-30.

46 Walsh T, Casadei S, Lee MK, Pennil CC, Nord AS, Thornton AM, et al. Mutations in 12 genes for inherited ovarian, fallopian tube, and peritoneal carcinoma identified by massively parallel sequencing. Proc Natl Acad Sci U S A 2011; 108: 18032-7.

47 Konstantinopoulos PA, Spentzos D, Karlan BY, Taniguchi T, Fountzilas $\mathrm{E}$, Francoeur N, et al. Gene expression profile of BRCAness that correlates with responsiveness to chemotherapy and with outcome in patients with epithelial ovarian cancer. J Clin Oncol 2010; 28: 35556.

48 Saal LH, Gruvberger-Saal SK, Persson C, Lövgren K, Jumppanen $\mathrm{M}$, Staaf J, et al. Recurrent gross mutations of the PTEN tumor suppressor gene in breast cancers with deficient DSB repair. Nat Genet 2008; 40: 102-7.

49 Forster MD, Dedes KJ, Sandhu S, Frentzas S, Kristeleit R, Ashworth A, et al. Treatment with olaparib in a patient with PTEN-deficient endometrioid endometrial cancer. Nat Rev Clin Oncol 2011; 8: $302-$ 6.

50 Joost $\mathrm{P}$, Bendahl PO, Halvarsson B, Rambech E, Nilbert M. Efficient and reproducible identification of mismatch repair deficient colon cancer: validation of the MMR index and comparison with other predictive models. BMC Clin Pathol 2013; 13: 33.

51 Byrski T, Huzarski T, Dent R, Marczyk E, Jasiowka M, Gronwald J, et al. Pathologic complete response to neoadjuvant cisplatin in BRCA1positive breast cancer patients. Breast Cancer Res Treat 2014; 147: 401-5.

This work is licensed under the Creative Commons Attribution-NonCommercial-No Derivative Works 3.0 Unported License. To view a copy of this license, visit http://creativecommons.org/licenses/by-nc$\mathrm{sa} / 3.0 /$ 